\title{
A New Scale for the Evaluation of Proactive and Retroactive Interference in Mild Cognitive Impairment and Early Alzheimer's Disease
}

Rosie E Curiel ${ }^{1}$, Elizabeth Croccoํ, Amarilis Acevedo², Ranjan Duara ${ }^{3}$, Joscelyn Agron ${ }^{3}$ and David A Loewenstein ${ }^{1 *}$

${ }^{1}$ Center on Aging and Department of Psychiatry and Behavioral Sciences, Miller School of Medicine, University of Miami, Florida, USA

${ }^{2}$ Center for Psychological Studies, Nova Southeastern University, Davie, Florida, USA

${ }^{3}$ Wien Center for Alzheimer's Disease and Memory Disorders, Mount Sinai Medical Center, Florida, USA

\begin{abstract}
Objective: The authors evaluated the psychometric properties and clinical utility of the Loewenstein-Acevedo Scale for Semantic Interference and Learning (LASSI-L), in patients with amnestic Mild Cognitive Impairment (aMCI) and early Alzheimer's disease (AD).

Methods: Subjects were administered Target List A and instructed to remember 15 common words belonging to a specific semantic category, using multi-modal, active encoding procedures. After free recall and cued recall trials of the target list, a second learning trial was offered, followed by a cued recall trial, to facilitate the initial acquisition of targets. Thereafter, the subject was exposed to a semantically-related List B, which was administered in the same manner as Target List A. Test-retest reliability, concurrent and discriminative validity were assessed. LASSI-L measures were then correlated with Magnetic Resonance Imaging (MRI) measurements of medial temporal lobe atrophy (MTA).

Results: High test-retest, concurrent and discriminative validity was obtained for LASSI-L subscales, and MTA atrophy scores were highly and negatively correlated with LASSI-L indices.

Conclusion: Subtests of the LASSI-L demonstrate high reliability and validity, and are strongly associated with MRI biomarkers of early neurodegenerative disease. It is concluded that the LASSI-L is a highly promising test for the assessment of mild cognitive impairment and early $A D$ among the elderly.
\end{abstract}

Keywords: Alzheimer's; Mild cognitive imparment; Neuropsychological assessment; Memory semantic interference; Proactive interference

\section{Introduction}

Neuropsychological screening and diagnostic measures are typically relied upon to identify the earliest stages of cognitive and functional impairment, associated with neurodegenerative disease processes, such as Alzheimer's disease (AD). Traditional neuropsychological measures, initially developed for the assessment of dementia, are often not well suited to identify the subtle changes in cognition, that manifest during the earliest stages of disease. As such, more sensitive neuropsychological assessment measures are required to identify the earliest neurocognitive deficits associated with $\mathrm{AD}$, and other neurodegenerative disorders [1].

Amnestic Mild Cognitive Impairment (aMCI) is now commonly accepted to reflect a prodrome of Alzheimer's disease [2]. This has led to a greater focus on employing measures in clinical settings that are more sensitive to the presence of MCI. While delayed recall and rate of forgetting had been previously considered the hallmark of medial temporal lobe dysfunction in $\mathrm{AD}$, it has become increasingly recognized that deficits in measures of initial learning may be as sensitive, as or more sensitive than delayed recall in the identification of MCI [3,4]. Some researchers propose that the semantic deficit in Alzheimer's disease reflects a degradation of the semantic network itself $[5,6]$, while others attribute the deficit to impaired retrieval from the network [7]. Mildly impaired AD patients are specifically prone to semantic intrusions that suggest incomplete processing of target stimuli [8]. To explore this susceptibility that AD patients had in making semantic errors, Loewenstein and Acevedo [9] developed an interference paradigm, in which semantically similar objects competed for expression in memory. Their aim was to further highlight the specific information processing deficits associated with $\mathrm{AD}$, and help to identify those in the early stages of the disorder (i.e. MCI). This work demonstrated those with MCI attributable to early AD had significant deficits with proactive interference, when two semantically related lists of targets had to be learned and recalled.

A major limitation of these existing studies is that they fail to account for differences in initial recall, and employ passive rather than active encoding of to-be-remembered information (e.g. CVLT-II, Logical Memory for passages). Further, the evaluation of interference effects based on a limited number of shared semantic categories, without specifying the shared category at the time initial encoding (i.e. furniture on the CVLT-II), is also a limitation of routinely employed paradigms. Active encoding refers to the process by which the examiner may facilitate strategy use, in this case, directly providing the shared semantic categories of to-be-remembered words. Active encoding has been found to be an important element in assuring proper processing of information to be recalled [10,11]. Furthermore, we have previously proposed that multi-modal encoding of common objects presented across various learning trials, can optimize performance [12]. In addition, multi-modal encoding using vision, touch and auditory processing may be more culturally and educationally fair $[13,14]$.

*Corresponding author: David A Loewenstein, Center on Aging and Departmen of Psychiatry and Behavioral Sciences, Miller School of Medicine, University of Miami, 1695 NW 9th Avenue, Suite 3208G, Miami Beach, Florida, 33136, USA, Tel: (305) 355-9028; E-mail: DLoewenstein@med.miami.edu

Received February 20, 2013; Accepted March 28, 2013; Published April 02, 2013

Citation: Curiel RE, Crocco E, Acevedo A, Duara R, Agron J, et al. (2013) A New Scale for the Evaluation of Proactive and Retroactive Interference in Mild Cognitive Impairment and Early Alzheimer's Disease. Aging Sci 1: 102. doi: 10.4172/23298847.1000102

Copyright: @ 2013 Curiel RE, et al. This is an open-access article distributed under the terms of the Creative Commons Attribution License, which permits unrestricted use, distribution, and reproduction in any medium, provided the original author and source are credited. 
Routinely employed clinical measures are limited in capturing the subtle changes inherent early in the AD disease process. Specifically, the limitations of current learning and memory paradigms is that they do not facilitate active encoding of targets, semantic relatedness of the targets are assumed, cued recall is not employed, and initial level of retrieval deficits is not accounted for. These variables can affect overall performance, as well as impact interference measures.

To address these concerns, we developed a validated paradigm called the Loewenstein-Acevedo Scales of Semantic Interference and Learning (LASSI-L) [9] (Appendix A). The LASSI-L addresses the aforementioned difficulties in previous studies by: a) explicitly identifying to the person, the semantic categories around which learning should be organized at the time that the target words are initially presented, instead of specifying the categories after the initial free recall trials are completed (such as is currently done on other widely used measures, such as the CVLT-II); b) using a second list in which every to-be-remembered target is semantically related to targets on the first list; c) more active encoding of information to be remembered by increasing depth of initial processing; d) evaluating free recall versus the use of semantic cues at the end of retrieval trials; and e) allowing for the adjustment of initial memory strength, in evaluating the ability to benefit from semantic cues and susceptibility to proactive and retroactive interference.

The purpose of the current investigation was to determine the reliability and validity of the LASSI-L in the assessment of MCI and early AD. Further, we attempted to determine the extent to which medial temporal atrophy on MRI was related to performance on different LASSI-L measures.

\section{Methods}

\section{Subjects}

Subjects were recruited from a study investigating longitudinal changes associated with mild cognitive impairment and normal aging, as well as from the memory disorders clinic at the Wien Center for Alzheimer's Disease and Memory Disorders at Mount Sinai Medical Center, as described below. The data included in this manuscript was obtained in compliance with the internal review board of Mt. Sinai Medical Center and the University of Miami. Subjects diagnosed with amnestic mild cognitive impairment (aMCI) met Petersen's [15] criteria. These include a memory complaint by the patient, and preferably an informant, objective memory deficits on clinical evaluation, and cognitive deficits not sufficient to interfere with social, and/or occupational function by DSM-IV criteria. All of these subjects obtained a global Clinical Dementia Rating Score (CDR) [16] of 0.5, equivalent to MCI. Subjects classified as probable AD met DSM-IV (35) criteria for dementia and NINCDS-ADRDA (36) criteria for probable Alzheimer's disease (AD). Forty-four subjects were normal elderly (NE) participants, with no evidence of cognitive impairment on clinical examination, had a score of 27 or above in the Folstein Mini-Mental State (MMSE) [17], and no scores one half standard deviation or lower on a three-trial version of the Fuld Object Memory Evaluation [18].

\section{Procedures}

The LASSI-L instructs a person to remember a list of 15 common words that are fruits, musical instruments or articles of clothing (five words per category). The person is asked to read the words for the target list out loud as each is presented individually at 4 -second intervals. The words are printed using a 48-size font. In the unlikely event that the person cannot correctly read the word, the word is read by the examiner, and the person is asked to repeat the word. If a person does not know the meaning of one of the words (also unlikely), the examiner tells the person what category the word belongs to (e.g. "Lime is a fruit"), and the person is asked to repeat the word. After the person has read all 15 words, they are then asked to recall the words. After free recall has ended, they are presented with each category cue (e.g. clothing), and asked to recall the words that belonged to that category. They are then presented with target List A items for a second learning trial. Thereafter, they are then provided semantic cues and asked to recall the items that belonged to each category. This second category-cued learning and category-cued recall trials strengthen the initial acquisition of List A targets. Exposure to the semantically related list (i.e. List B) is then conducted in the same manner as exposure to List A. List B consists of 15 words, 5 of which belong to each of the three categories used in List A (i.e. fruits, musical instruments and articles of clothing). Following the presentation of the List B words, the person is asked to free recall the List B words, assessing proactive interference effects. Then, each category cue is given, and they are asked to recall List B words that belonged to each of the categories. List B words are presented again, followed by a second category-cued recall trial. Finally, to assess retroactive interference, they are asked to free recall the original List A words. This is followed by a category-cued recall trial of List A words. The exposure time for each word in all learning trials of Lists A and B is 4 seconds per word. The maximum allotted time for each free recall trial of Lists A and B is 60 seconds, and the maximum allotted time for cued recall of each of the individual categories for Lists A and B is 20 seconds.

\section{Magnetic Resonance Imaging (MRI) of the medial temporal lobe}

Structural brain MRIs were obtained on a 1.5 Tesla MRI machine, using proprietary 3-D MPRAGE (Siemens) sequences, to acquire contiguous coronal slices of $1.5 \mathrm{~mm}$ or less in thickness. Structural MRIs were then constructed in the coronal plane, perpendicular to the anterior commissure-posterior commissure line. Visual rating scale software [19,20], with high reliability and validity was used [21], to standardize blind ratings of atrophy in the left and right hippocampus (HPC), entorhinal cortex (ERC) and perirhinal cortex (PRC). The visual rating scale is used to assess a standard coronal slice intersecting the mammillary bodies. Medial temporal atrophy (MTA) is evaluated by assessing atrophy in three distinct areas on each hemisphere: the hippocampus, entorhinal cortex and perirhinal cortex. Ratings were then conducted based on a five point scale, with "0" signifying no atrophy and "4" signifying the most severe atrophy. Hemispheric ratings for each hemisphere were calculated by taking the sum of the ratings for the HPC, ERC and PRC on each side.

\section{Results}

\section{Test-retest reliability}

Fifteen elders ( 10 males and five females), aged 65 to 89 years (mean age $=76.7, \mathrm{SD}=6.0$ years) were diagnosed with amnestic MCI (aMCI) by Petersen's criteria [15]. The mean MMSE scores for this group was $26.1(\mathrm{SD}=2.7)$, and the suspected clinical etiological diagnosis based on clinical evaluation was MCI attributable to Alzheimer's Disease [22]. All aMCI subjects were administered the LASSI-L on two occasions, within a 18-week interval (Mean=8.0; $\mathrm{SD}=6.6$ weeks). Test-retest comparisons were conducted for free recall of Lists A and B, as well as Short-Delay free recall for List A. In addition, test-retest reliabilities were established for first and second cued recall of List A, the first and second cued recall of List B (vulnerable to proactive interference), and the final cued recall 
Citation: Curiel RE, Crocco E, Acevedo A, Duara R, Agron J, et al. (2013) A New Scale for the Evaluation of Proactive and Retroactive Interference in Mild Cognitive Impairment and Early Alzheimer's Disease. Aging Sci 1: 102. doi: 10.4172/2329-8847.1000102

of List A (vulnerable to retroactive interference). One-tailed Pearson Product Moment Correlation Coefficients were obtained because of the directional hypotheses concerning test-retest relationships. High, statistically significant test-retest reliabilities were obtained for Free Recall List A ( $\mathrm{r}=.55 ; \mathrm{p}<.017)$; Cued List A2 ( $\mathrm{r}=.70 ; \mathrm{p}<.003)$, Free Recall List $\mathrm{B}(\mathrm{r}=.51 ; \mathrm{p}<.03)$; Cued List B1 $(\mathrm{r}=.62 ; \mathrm{p}<.009)$, Cued List $\mathrm{B} 2(\mathrm{r}=.80$; $\mathrm{p}<.001)$, and Delayed Cued Recall List $\mathrm{A}(\mathrm{r}=.66 ; \mathrm{p}<.005)$. Indices for the stability of the List A1 cued recall $(\mathrm{r}=.40 ; \mathrm{p}>.08)$ and short free recall $(\mathrm{r}=.32 ; \mathrm{p}>.13)$ of List A were not statistically significant.

Nineteen $\mathrm{AD}$ patients (10 males and 9 females), 70 to 86 years (mean age $=81.4, \mathrm{SD}=5.1$ years), were diagnosed as having probable AD by NINCDS-ADRDA criteria [23]. The mean MMSE scores for this group was $20.71(\mathrm{SD}=3.9)$. These subjects were administered the LASSI-L on two occasions within a 20 -week interval (Mean=6.9; $\mathrm{SD}=5.8$ weeks). High statistically significant test-retest reliabilities were obtained for Free Recall List A ( $\mathrm{r}=.59 ; \mathrm{p}<.004)$, Cued List B1 ( $\mathrm{r}=.58$; $\mathrm{p}<.005)$, Cued List B2 ( $\mathrm{r}=.65 ; \mathrm{p}<.001)$, and Short-Delay Free Recall List A $(r=.53 ; \mathrm{p}<.01)$. Cued List A1 $(\mathrm{r}=.39 ; \mathrm{p}=.05)$ and Free Recall List B $(\mathrm{r}=.39 ; \mathrm{p}<.05)$ were statistically significant, but the obtained correlation coefficients were relatively modest. Cued Recall List A2 ( $r=.37 ; \mathrm{p}>.059)$ and Short Delay Cued Recall List A $(r=.23 ; \mathrm{p}>.17)$ were not statistically significant.

\section{Discriminative validity}

We performed discriminative validity studies on the 15 aMCI subjects who had participated in test-retest reliability studies, described above. In addition, 19 patients aged 70 to 86 years (mean age $=76.7,6.0$ years), who were demented and met NINCDS-ADRDA [23] criteria for a diagnosis of probable $\mathrm{AD}$, were also evaluated. $53 \%$ of these $\mathrm{AD}$ participants were male and $68 \%$ of the sample spoke English as their primary language. $32 \%$ of these individuals spoke Spanish as their primary language. The mean MMSE score for this group was 20.7 $(\mathrm{SD}=3.9)$. We established discriminative validity by contrasting their performance with 44 normal elderly control subjects.

As indicated in table 1, all aMCI participants scored lower on all indices of the LASSI-L, as compared to normal elderly subjects. Demented Probable AD participants also scored lower on all of the LASSI-L measures, relative to normal elderly subjects. Finally, aMCI subjects scored lower than Probable AD subjects on all free and cued recall List A trials. In comparing aMCI participants to normal elderly subjects, step-wise logistic regression analyses revealed an overall correct classification rate of $88 \%$ (73.3\% sensitivity, $93.2 \%$ specificity) (Table 2), with the second List A recall and the first List B recall entering into the equation. For Probable AD vs. normal elderly comparisons, an overall correct classification rate of $92.1 \%$ (78.9\% sensitivity and 97.7 $\%$ specificity) was obtained, with only the second List A recall entering into the equation.

\section{Concurrent validity}

We compared scores on the LASSI-L with those obtained using the Modified Fuld Object Memory Evaluation and the Total Interference Score of the Semantic Interference Test [12], for 15 aMCI or probable AD subjects, who were administered these measures. We hypothesized that free recall learning trials of the OME would most highly relate to free recall trials of the LASSI-L, while the Total Interference Score on the Semantic Interference Test would be most associated with cued recall scores on the List B and Delay List A, as well as Free Recall of List B (all susceptible to interference effects). Consistent with expectations, the Fuld OME total three trial score was significantly associated with List A Free Recall $(\mathrm{r}=.49 ; \mathrm{p}<.04)$ and List B Free Recall $(\mathrm{r}=.53 ; \mathrm{p}<.03)$, as well as the first List A Cued Recall $(\mathrm{r}=.46 ; \mathrm{p}<.05)$. The Total Interference score of the SIT was associated with List B1 and List B2 recall $(\mathrm{r}=.61$;

\begin{tabular}{|c|c|c|c|c|}
\hline & Normal Elderly (NE: N=44) & $\mathrm{aMCl}(\mathrm{N}=15)$ & Dementia (N=19) & F-Value $(2,116)$ \\
\hline Free recall list $A$ & $10.43^{\mathrm{a}}(\mathrm{SD}=2.5)$ & $6.53^{b}(S D=2.4)$ & $4.58^{\mathrm{c}}(\mathrm{SD}=2.4)$ & $41.27^{* * *}$ \\
\hline Cued recall 1 list $A$ & $11.53^{\mathrm{a}}(\mathrm{SD}=2.2)$ & $8.20^{b}(S D=1.3)$ & $6.53^{\mathrm{c}}(\mathrm{SD}=2.2)$ & $46.14^{* \star *}$ \\
\hline Cued recall 2 list $A$ & $13.89^{a}(\mathrm{SD}=1.3)$ & $11.07^{\mathrm{b}}(\mathrm{SD}=2.0)$ & $8.84^{\circ}(S D=1.9)$ & $69.25^{* * *}$ \\
\hline List $B$ free recall & $7.09^{\mathrm{a}}(\mathrm{SD}=2.6)$ & $5.40^{\mathrm{ab}}(\mathrm{SD}=2.1)$ & $3.79^{b}(S D=1.7)$ & $14.17^{\star \star \star}$ \\
\hline List B cued recall 1 & $8.05^{\mathrm{a}}(\mathrm{SD}=2.4)$ & $4.27^{\mathrm{b}}(\mathrm{SD}=2.5)$ & $4.12^{\mathrm{b}}(\mathrm{SD}=2.2)$ & $26.11^{* *}$ \\
\hline List B cued recall 2 & $11.32^{\mathrm{a}}(\mathrm{SD}=2.1)$ & $7.67^{\mathrm{b}}(\mathrm{SD}=1.3)$ & $6.37^{\mathrm{b}}(\mathrm{SD}=2.2)$ & $37.93^{* * *}$ \\
\hline $\begin{array}{l}\text { Short-delay recall list a free } \\
\text { recall }\end{array}$ & $6.75^{\mathrm{a}}(\mathrm{SD}=3.0)$ & $2.67^{\mathrm{b}}(\mathrm{SD}=2.4)$ & $1.95^{\mathrm{b}}(\mathrm{SD}=1.7)$ & $27.17^{\star * *}$ \\
\hline Short delay recall list a cued recall & $8.70^{a}(S D=2.8)$ & $6.33^{b}(S D=2.6)$ & $5.37^{\mathrm{b}}(\mathrm{SD}=1.7)$ & $13.39^{* * *}$ \\
\hline
\end{tabular}

Note: 1$\left.)^{* * *} p<.001\right) ; 2$ ) All other LASSI-L indices are adjusted for scores on initial free recall for List A with Tukey HSD Tests conducted at $p \leq .05$

Table 1: LASSI-L performance among different diagnostic groups.

\begin{tabular}{|c|c|c|c|c|c|}
\hline & & & \multicolumn{3}{|c|}{ Predicted } \\
\hline & \multirow{2}{*}{\multicolumn{2}{|c|}{ Observed }} & \multicolumn{2}{|c|}{ Actual Diagnosis } & \multirow{2}{*}{ Percentage Correct } \\
\hline & & & NE & $\mathrm{aMCl}$ & \\
\hline \multirow{3}{*}{ Step 1} & \multirow{2}{*}{ Predicted diagnosis based } & NE & 41 & 3 & 93.2 \\
\hline & & $\mathrm{aMCl}$ & 6 & 9 & 60.0 \\
\hline & \multicolumn{2}{|c|}{ Overall percentage } & & & 84.7 \\
\hline \multirow{3}{*}{ Step 2} & \multirow{2}{*}{ Predictor of diagnosis } & $\mathrm{NE}$ & 41 & 3 & 93.2 \\
\hline & & $\mathrm{aMCl}$ & 4 & 11 & 73.3 \\
\hline & \multicolumn{2}{|c|}{ Overall percentage } & & & 88.1 \\
\hline
\end{tabular}

a. The cut value is .500; The predictor on Step 1 was List A (Second recall), while in Step 2, incremental predictive power was obtained by adding List B (First recall) to List A (Second recall).

Table 2: Sensitivity and specificity of LASSI-L measures to distinguish aMCI from NE subjects. 
Citation: Curiel RE, Crocco E, Acevedo A, Duara R, Agron J, et al. (2013) A New Scale for the Evaluation of Proactive and Retroactive Interference in Mild Cognitive Impairment and Early Alzheimer's Disease. Aging Sci 1: 102. doi: 10.4172/2329-8847.1000102

Page 4 of 5

\begin{tabular}{|c|c|c|c|c|c|c|c|c|}
\hline & List A free recall List A1 cued recall & List A2 cued recall & List B free recall & List B1 cued recall & List B2 cued recall & Short Delay List A free recall & Short Delay List A cued recall \\
\hline MTA Left & -.43 & -.43 & $-.53^{*}$ & -.47 & $-.75^{\star * *}$ & $-.78^{* * *}$ & -.07 \\
\hline MTA Right & -.36 & -.40 & $-.57^{\star}$ & -.33 & $-.58^{*}$ & $-.65^{* *}$ & -.04 \\
\hline
\end{tabular}

${ }^{*} \mathrm{p}<.05 ; \mathrm{P}<.01 ; \mathrm{p} \leq .001$

Table 3: Correlations between measures of medial temporal lobe atrophy and LASSI-L measures.

$\mathrm{p}<.009$ and $\mathrm{r}=.66 ; \mathrm{p}<.005)$, Free Recall List $\mathrm{B}(\mathrm{r}=.63 ; \mathrm{p}<.007)$ and Free Recall List A $(\mathrm{r}=.49 ; \mathrm{p}<.04)$.

\section{Lassi-l indices and measures of medial temporal atrophy}

We examined the MRI scans of 16 individuals $(7$ males and 9 females) diagnosed with mild cognitive impairment, but who met all other criteria for an NINCDS-ADRDA diagnosis of probable AD [23], or probable $\mathrm{AD}$ patients with a mild dementia. The mean age of these subjects was 79.44 years $(\mathrm{SD}=6.5$; range $=67-92)$, the mean level of education was 13.19 years $(\mathrm{SD}=4.9$; range $=4-25)$, and the mean MMSE score was $24.38(\mathrm{SD}=3.8$; range=16-30). $69 \%$ of the sample spoke English as their primary language, while $31 \%$ spoke Spanish as their primary language. All subjects had MRI scans no more than one year from their initial LASSI-L evaluation. The average test-retest interval was 27.06 weeks $(\mathrm{SD}=16.2$ : range $=2-52$ weeks).

For both the right and left hemisphere, a medial atrophy temporal score was determined by summing atrophy ratings for the hippocampus, entorhinal cortex and perirhinal cortex. Higher MTA scores signified greater levels of atrophy. These scores were then examined, with respect to their associations with LASSI-L measures. As depicted in table 3, left hemisphere and MTA atrophy scores were negatively correlated with second cued recall of List A1 ( $\mathrm{r}=-.53 ; \mathrm{p}<.04)$, first cued recall of List $\mathrm{B}$ $(\mathrm{r}=-75 ; \mathrm{p} \leq .001)$ and second cued recall of List $\mathrm{B}(\mathrm{r}=-78 . ; \mathrm{p} \leq .001)$. There were no statistically significant associations between left MTA and first trial recall of List A ( $\mathrm{r}=-.43 ; \mathrm{p}>.09)$, short-delay recall of List A ( $\mathrm{r}=.04$; $\mathrm{p}>.87)$, first free recall of List $\mathrm{A}(\mathrm{r}=-.43 ; \mathrm{p}>.09)$ and second free recall of List $\mathrm{B}(\mathrm{r}=-.47 ; \mathrm{p}>.06)$. With regard to the right hemisphere, MTA atrophy scores were negatively correlated with second cued recall of List A1 ( $\mathrm{r}=-.57 ; \mathrm{p} \leq .02)$, first cued recall of List $\mathrm{B}(\mathrm{r}=-58 ; \mathrm{p}<.02)$ and second cued recall of List $B(r=-65 . ; p<.01)$. There were no statistically significant associations between right MTA and first trial recall of List $\mathrm{A}(\mathrm{r}=-.36 ; \mathrm{p}>.16)$, short-delay recall of List A $(\mathrm{r}=-.14 ; \mathrm{p}>.63)$, first free recall of List $\mathrm{A}(\mathrm{r}=-.37 ; \mathrm{p}>.16)$ and free recall of List $\mathrm{B}(\mathrm{r}=-.33 ; \mathrm{p}>.20)$.

\section{Discussion}

The present study was designed to examine the psychometric properties of the LASSI-L, a newly developed instrument for the assessment of learning, and semantic interference in individuals with mild cognitive impairment and early AD. The LASSI-L is unique, in that it explicitly and from the outset, identifies the precise semantic categories around which learning should be organized, promotes more active encoding of information, to be remembered by increasing depth of initial processing; and focuses on cued recall and vulnerability to semantic interference.

The current investigation evidenced that most subscales of the LASSI-L had good test-retest reliability for aMCI and Mild AD patients. In addition, performance on list learning tests such as the Fuld Object Memory Evaluation was most highly associated with free recall measures on the LASSI-L, while the interference measure of the Semantic Interference Test highly correlated with LASSI-L List B free and cued recall measures; the ones most susceptible to proactive semantic interference.

Scores on all LASSI-L measures were higher for cognitively normal elderly subjects, relative to aMCI subjects and AD patients with dementia. In addition, high levels of discriminative validity were obtained in differentiating aMCI from cognitively normal groups based upon logistic regression analyses. Finally, MRI measures of medial temporal lobe atrophy were highly associated with cued recall for both List A and List B, but was not associated with free recall measures. Uniformly, the highest associations were obtained between medial temporal atrophy (MTA) and List B cued recall measures; the LASSI-L learning trials most susceptible to proactive semantic interference. Interestingly, earlier findings have demonstrated that vulnerability to semantic interference is one of the early changes in aMCI patients, which is likely a reflection of an early AD process [12]. In fact, susceptibility to proactive interference was one of the strongest predictors of progression of aMCI to AD dementia, over a two to three year period [19]. The finding that MTA was associated with cued recall rather than free recall is particularly noteworthy and consistent with the notion that cued recall deficits may be more sensitive markers of $\mathrm{AD}$, than measures of free recall [11].

A potential limitation of the current study is that we employed modest samples, and that these findings need to be generalized to different patient groups in different settings. It might also be potentially argued that simply providing the three categories around which to-beremembered information might be initially organized might guarantee that every subject will initially encode information in that manner. However, our goal was to have an equal category representation in the beginning, middle and end of the to-be-remembered list, and to provide a mechanism by which a superordinate category (fruits, musical instruments, articles of clothing) was present to better facilitate active encoding. The importance of these superordinate categories were further reinforced by using these same categories for cued recall after every learning trial, which we believe best facilitated investigation of semantic interference effects.

Although the results of this study are preliminary, our findings indicate that the LASSI-L has acceptable properties of reliability and validity, and the instrument holds promise as a diagnostic tool that can be used by clinicians for identifying early cognitive manifestations of AD. Further investigations will be required to determine the utility of the LASSI-L in other memory impaired populations, as well as predicting the trajectory of cognitive decline over time. The current study suggests that the instrument is worthy of further research.

\section{Acknowledgements}

The authors hold no financial or other relationships that could be interpreted as a conflict of interest affecting this manuscript. The authors are full time faculty members of the University of Miami Miller School of Medicine, Nova Southeastern University and Mt. Sinai Medical Center.

\section{References}

1. Brooks LG, Loewenstein DA (2010) Assessing the progression of mild cognitive impairment to Alzheimer's disease: Current trends and future directions. Alzheimers Res Ther 2: 28

2. Dubois B, Feldman HH, Jacova C (2007) Research criteria for the diagnosis of Alzheimer's disease: Preliminary findings. Am J Geriatr Psychiatry 11: 252-255.

3. Greenaway MC, Lacritz LH, Binegar D, Weiner MF, Lipton A, et al. (2006) Patterns of verbal memory performance in mild cognitive impairment Alzheimer's disease and normal aging. Cogn Behav Neurol 19: 79-84. 
Citation: Curiel RE, Crocco E, Acevedo A, Duara R, Agron J, et al. (2013) A New Scale for the Evaluation of Proactive and Retroactive Interference in Mild Cognitive Impairment and Early Alzheimer's Disease. Aging Sci 1: 102. doi: 10.4172/2329-8847.1000102

4. Loewenstein DA, Acevedo A, Schram L (2003) Semantic interference in mild Alzheimer's disease: revising the NINCDS-ADRDA criteria. Lancet Neurol 6 734-746.

5. Hodges JR, Salmon DP, Butters N (1992) Semantic memory impairment in Alzheimer's disease: failure of access or degraded knowledge? Neuropsychologia 30: 301-314

6. Rogers SL, Friedman RB (2008) The underlying mechanisms of semantic memory loss in Alzheimer's Disease and Semantic Dementia. Neuropsychologia 46: $12-21$.

7. Nebes RD (1992) Cognitive dysfunction in Alzheimer's disease. The handbook of aging and cognition. Hillsdale NJ, Lawrence Erlbaum Associates, Inc., England, UK 373-446.

8. Loewenstein DA, D'Elia L, Guterman A, Eisdorfer C, Wilkie F, et al. (1991) The occurrence of different intrusive errors in patients with Alzheimer's Disease multiple cerebral infarctions and major depression. Brain Cogn 16: 104-117.

9. Loewenstein DA, Acevedo A (2005) Loewenstein acevedo scale for semantic interference and learning: manual of procedures and interpretation. Miam Beach, Florida.

10. Buschke H, Sliwinski M, Kuslansky G, Lipton RB (1995) Aging, encoding specificity and memory change in the Double Memory Test. J Int Neuropsycho Soc 1: 483-493.

11. Buschke H, Sliwinski M, Kuslansky G, Lipton RB (1997) Diagnosis of early dementia by the Double Memory Test: Encoding specificity improves diagnostic sensitivity and specificity. Neurology 48: 989-997.

12. Loewenstein DA, Acevedo A, Luis CA, Crum T, Barker WW, et al. (2004) Semantic interference deficits and the detection of mild Alzheimer's Disease and mild cognitive impairment without dementia. J Int Neuropsychol Soc 1: 91-100.

13. Loewenstein DA, Ardila A, Rosselli M, Hayden S, Duara R, et al. (1992) A comparative analysis of functional status among Spanish- and Englishspeaking patients with dementia. J Gerontol 47: P389-P394.
14. Loewenstein DA, Arguelles T, Barker WW, Duara R (1993) A Comparative analysis of neuropsychological test performance of spanish-speaking and english-speaking patients with Alzheimer's Disease. J Gerontol 48: P142-P149.

15. Petersen RC, Doody R, Kurz A, Mohs RC, Morris JC, et al. (2004) Current concepts in mild cognitive impairment. Arch Neurol 58: 1985-1992.

16. Morris JC (1993) The Clinical Dementia Rating (CDR): Current versions and scoring rules. Neurology 43: 2412-2414.

17. Folstein M, Folstein S, McHugh P (1975) Mini-mental state: A practical method for grading the cognitive state of patients for the physician. J Psychiatr Res 12 189-198.

18. Fuld PA (1981) Fuld object-memory evaluation. Stoelting Co, Illinois.

19. Duara R, Loewenstein DA, Potter E, Appel J, Greig M, et al. (2008) Medial temporal lobe atrophy on MRI scans and the diagnosis of Alzheimer's Disease. Neurology 71: 1986-1992.

20. Shen Q, Loewenstein DA, Potter E, Zhao JW, Appel J, et al. (2011) Volumetric and visual rating of MRI scans in the diagnosis of Amnestic MCl and Alzheimer's Disease. Alzheimers Dement 7: e101-e108.

21. Urs R, Potter E, Barker WW, Appel J, Loewenstein DA, et al. (2009) Visua rating system for assessing magnetic resonance images: $A$ tool in the diagnosis of mild cognitive impairment and Alzheimer Disease. J Comput Assist Tomogr 33: 73-78.

22. Albert MS, DeKosky ST, Dickson D, Dubois B, Feldman HH, et al. (2011) The diagnosis of mild cognitive impairment due to Alzheimer's disease: recommendations from the National Institute on Aging-Alzheimer's Association workgroups on diagnostic guidelines for Alzheimer's disease. Alzheimers Dement 7: 270-279.

23. McKhann G, Drachman D, Folstein M, Katzman R, Price D, et al. (1984) Clinical diagnosis of Alzheimer's disease: report of the NINCDS-ADRDA Work Group under the auspices of the Department of Health and Human Services Task Force on Alzheimer's disease. Neurology 34: 939-944 